\title{
Study on Hazard Factors and Preventive Measures of Zoonotic Disease in Earthquake Area
}

\author{
Chen Liyan \\ ${ }^{1}$ College of Animal Science and Technology, Inner Mongolia University for the Nationalities, \\ Tongliao 028042,China. \\ chenliyan1010@163.com
}

\begin{abstract}
Objective: After earthquake occurring in pastoral areas, due to the reasons such as many animals are dead, drinking water is polluted, the condition of food hygiene is poor, the weather is hot and the other factors, it is extremely easy to occur anthropozoonosis. The purpose of this study is to prevent the occurrence of anthropozoonosis in earthquake area. Method: Through collecting the information in the disaster area, implementing the emergency action and other aspects to carry out the anthropozoonosis prevention and control work under the post-earthquake emergency in the paper according to the veterinarian's work in the earthquake relief work. Result: Three stages of epidemic prevention are established in the paper according to the information collection and analysis result in the earthquake affected area, the planning and deployment of epidemic prevention work are conducted according to the features at these three stages. Conclusion: The prevention and control of anthropozoonosis in the earthquake disaster area can guarantee the life health of the people in the disaster area, it is also an important content of animal health work, and the experience in the research is vital for the healthy survival and life of the people and animal after the disaster.
\end{abstract}

Keywords: Earthquake Areas, anthropozoonosis, prevention and control

\section{Introduction}

On April 14, 2010, six earthquakes occurred in Yushu County, Yushu Tibetan Autonomous Prefecture, Qinghai Province with the highest magnitude of 7.1 level, and the earthquake epicenter was near the county. The paralysis of disease surveillance and control system were caused by the earthquake, the conditions conducive to the occurrence and spread of infectious diseases were increased, therefore, the most important content in the earthquake relief work was preventing the outbreak of anthropozoonosis. After the disaster, the veterinarian department actively devoted in the epidemic prevention work, and it showed that the veterinarian played an important role in the prevention and control work of anthropozoonosis after the disaster[1-3]. In fact, the veterinarian participated in the earthquake relief work of Indian Ocean Tsunami and Kashmir Earthquake in 2005. How to prevent and control the anthropozoonosis work for the veterinarian was analyzed in the paper via the epidemic prevention information after the earthquake in Yushu.

\section{Preparation}

Seen from the epidemiology, there is the possibility to break out the epidemic disease at the first day after the earthquake, more and more excavated dead bodies and inventoried animal products need to be disinfected immediately, and the veterinarian shall provide the rapid and large-scale epidemic prevention action within the limited time under the extreme conditions. Only there is the 
in-advanced plan can we copy with the disaster successfully. Therefore, the country shall establish the veterinarian emergency organization within the veterinarian service system. It shall have the clear objective, and the action can be implemented at the occurrence of any emergency event relying on the daily preparation and rehearsal. It shall be composed by the veterinarian with the similar emergency epidemic prevention working experience or received the emergency training. In order to improve the working efficiency, the emergency organization shall absorb the veterinarian familiar with the local conditions to be the members.

\section{Measures adopted in the epidemic area after the earthquake}

After the earthquake, the veterinarian emergency organization shall copy with the potential hazardous pathogens for the people and animal according to the emergency plan, and reduce the harm to the personnel, society and economy to the greatest degree.

Information collection and analysis

Once the earthquake occurs, the veterinarian emergency budget shall be activated immediately, the information related with the anthropozoonosis epidemiology shall be collected as much as possible firstly and simply to facilitate to evaluate the risk of the outbreak of epidemic situation, judge the influence of the earthquake on the spread of disease preliminary, actively adjust and perfect the emergency budget, determine the needed resources and effective working strategy.

The earthquake was happened in Jiegu Town, Yushu County- the state capital of Yushu State, and $90 \%$ of the local residents' houses were collapsed. It is understood that most of the local people had not got up, and the casualty was serious. The earthquake occurred in Yushu Tibetan Autonomous Prefecture led to the death of at least 400 people and the injury of 8000 people. The fixed telephone communication in Yushu County was interrupted, and the local civil structure houses are collapsed seriously. The crack occurred in a reservoir in the earthquake area, and the related working staff adopted the water drainage and other emergency disposal measures. Currently, most victims only lived in the tents or housing cover temporarily, and they were faced with the crowded, poor health, mosquitoes and other problems; The great loss of (veterinarian) public health institutions was caused in the earthquake, and the epidemic reporting system in some regions was paralyzed basically. The veterinarian institutions at the levels in Qinghai earthquake area lost 1.64 billion yuan. The animal epidemic disease prevention and control system was paralyzed completely in Yushu region. It was damp rainy season in April with rising temperature and increased mosquitoes, the animals lost their homes were wandering at that time, some animals ate the dead body, attacked the people and became the disseminator of pathogen; some disaster areas had the rabies, anthrax, brucellosis and other anthropozoonosis epidemics; The storm, Barrier Lake and continuous aftershocks changed the ecological environment and created the external conditions conducive to the outbreak of disease.

The anthropozoonosis outbreak risk evaluation can evaluate the infectious disease risk via the collected information, and 20 kinds of anthropozoonosises (anthrax, brucellosis, mycobacteria, plague, streptococcus disease, escherichia coli disease, botulism, gas gangrene, tetanus, rabies, avian influenza, epidemic encephalitis b, leptospira, toxoplasmosis, attached red cell disease, schistosomiasis, malaria, swine cysticercosis, echinococcosis, chlamydia) can be determined. They shall enter into the monitoring network in priority, and give priority for the immunization and disease media control work.

Epidemic prevention process

Work at the first stage

At the first stage of epidemic prevention, the veterinarian mainly carries out the work in the food and water source security, animal carcass and metamorphic animal origin material destruction, safe 
temporary residence building, injured animal concentration, caring or slaughtering, disinfection mosquito and restoring the early detecting/monitoring system. Providing the safe drinking water is the most important prevention measure after the earthquake, so the disinfection becomes the veterinarian's prior work, especially that on the animal carcass and animal origin food, for they are the food of harmful animals (rodent, saprophagous animal and stray dog), these animal will spread the residual resin everywhere, thus increasing the risk of the spread of epidemic disease. Meanwhile, the corruption process may pollute the surface water, or enter into the public food chain and cause the disease. When disposing the dead body, the veterinarian shall protect himself, equip with the protective equipment, prevent the direct contact, rationally compound the disinfectant, and correctly use the low-pressure spray to spray on the dead body. After the earthquake, the animals without the owner in some disaster areas can not be cared. Added with many events that stray dogs attack people, the government adopted the culling policy, even some wild animals were dead due to the insufficient supply. In addition to the traffic factor, the fact of insufficient veterinarian and material was also highlighted.

Work at the second stage

The hot summer was coming in the disaster region, the health form is not optimistic. Added with the anxiety and the lack of material, people and animal's physical function were decreased, therefore, the risk of the outbreak of anthropozoonosis was increased greatly. At that time, the veterinarian shall continue to strengthen the disinfection extermination work, assist to restore the working ability and contact in the animal epidemic disease prevention and control centers at each level in the disaster region, restore the meat inspection and food safety work[4]; provide the shelter, feed and water for the surviving livestock (poultry); provide the medicine, vaccine, disinfection medicine and pesticide for the disaster area, and start the surveillance work of anthropozoonosis epidemiology.

Work at reconstruction stage

In the reconstruction stage, we shall focus on the objective to fully restore the veterinarian institution and working system, and reduce the risk of the outbreak of anthropozoonosis to the greatest degree. The experts hold that 3 stages shall be experienced for the prevention and control of epidemic disease in the earthquake area: prevention and control stage of infectious disease in summer and fall, prevention and control stage of infectious disease in the winter and spring and comprehensive recovery stage within 3-5 years. The epidemic prevention is successful only after passing these three stages smoothly. The Costa Rica earthquake in 1991 led to the environmental change of mosquito breeding site, and the malaria cases increased sharply after a month; At the same year, the flood and earthquake occurred in India, while the plague was broke out at the second year, and the great panic was caused, so the prevention and control work in the reconstruction stage is a long-term task. It lies in that the disaster changes the ecological condition in the disaster area, and the new environment may be not conducive to the veterinarian public health safety; The loss of veterinarian and veterinary institutions in some regions is severe, and it needs a long time to fully recover the work. Meanwhile, the number of livestock in the disaster area is decreased sharply, the demand on the meat product and the recovery production of animal husbandry may lead to the illegal trade and cause the disease.

\section{Conclusion}

The prevention and control of anthropozoonosis in the earthquake disaster area can not only guarantee the people's life health in the area, but also it is an important content of animal's health work, the health survival of the animals after the disaster is very important for the victims to recover the normal life. In recent years, the demand on the veterinarian is increasing constantly in the natural 
disaster relief work in the countries. After the earthquake, the Ministry of Agriculture sent the veterinarian experts to the disaster area to guide the epidemic prevention work, and they provided the support for the prevention and control of anthropozoonosis in the disaster area. So far, no large-scale epidemic situation has broken out in the disaster area. Therefore, the prevention and control of anthropozoonosis after the disaster is not only the responsibility of the people's public health department, but also that of the veterinarian department, while the good coordination and communication between the departments can ensure the accuracy and timeliness of the information collection, epidemic situation monitoring and risk evaluation, and the scientific guiding of prevention and control work. In the earthquake, the collaborative cooperation among the Ministry of Agriculture, the Ministry of Health and General Logistics Ministry Epidemic Prevention Experts, the active action of International Fund for Animal Welfare and domestic volunteers create the starting of good communication between the veterinarian and other departments in the emergency rescue in our country, and greatly improve the working efficiency.

\section{Reference}

[1] Asokan G V, Asokan V, Fedorowicz Z, et al. Use of a systems approach and evidence - based One Health for zoonoses research[J]. Journal of Evidence - Based Medicine, 2011, 4(2): 62-65.

[2] Gao, MengXu, et al. "Spatial prediction and analysis of Himalayan marmot plague natural epidemic foci in China based on HJ-1 satellite data." Science China Earth Sciences 53.1 (2010): 8-15.

[3] Lu L, Ren Z, Yue Y, et al. Niche modeling predictions of the potential distribution of Marmota himalayana, the host animal of plague in Yushu County of Qinghai[J]. BMC public health, 2016, 16(1): 183.

[4] Anthamatten P, Hazen H. An introduction to the geography of health[M]. Routledge, 2012. 